\title{
The Convergence of Population Ageing with Climate Change
}

\section{Sarah Harper ${ }^{1}$}

Published online: 4 December 2019

(C) Springer Nature B.V. 2019

The Climate Emergency Declaration was first issued in 2016 by selected countries and other jurisdictions to set priorities to mitigate climate change, with some 1180 jurisdictions and governments posting a Climate Emergency Declaration by November 2019. This alerts us to the fact that population ageing is not occurring in a vacuum and that it is at the intersection of this trend with the other mega-trends of the twentyfirst century, that some of the most important research questions lie. In 2009 the World Economic Forum noted that 3 of the top 5 trends in terms of risk were economic and 4 of the 5 in terms of impact were economic. But by 2019, the same forum was stating that 3 of the top 5 trends in terms of risk and 4 of the 5 in terms of impact were environmental. The convergence of climate change with the demographic trend of population ageing is clearly both complicated (knowable, predictable and controllable) and complex (unknowable nor predictable and thus difficult to control via interventions).

The Intergovernmental Panel on Climate Change (IPCC) considers it likely that the rise in atmospheric temperature will reach $1.5{ }^{\circ} \mathrm{C}$ degrees within the next 35 years. The current century trajectory is towards a rise of around $3.2{ }^{\circ} \mathrm{C}$. One of the most severe long term climate impacts will be rising sea levels and increasing heat sinks. Coastal cities combine these two factors to make them particularly vulnerable. An estimated 800 million people in more than 570 coastal cities will be vulnerable to a sea-level rise of $0.5 \mathrm{~m}$ by 2050 . The risks of rising sea levels are often compounded by storm surges and increased rainfall intensity. While there are considerable uncertainties over the precise interactions of regional sea-level rise and patterns of urban demography and development, it is clear that Asia will be the worst-affected region as a result of a combination of hydrology and population density. Asia is home to four-fifths of the people who are expected to be flooded if there is a $3{ }^{\circ} \mathrm{C}$ rise in global temperatures. In addition, $70 \%$ of the largest cities in Europe have areas vulnerable to rising sea levels. Africa has at

Sarah Harper

sarah.harper@ageing.ox.ac.uk

1 Oxford Institute of Population Ageing, University of Oxford, Oxford, UK 
least 19 vulnerable coastal cities with a population of more than 1 million, and half the population of northern Africa alone live in coastal cities. Deltas are home to more than two-thirds of the world's largest cities and 340 million people. These delta cities are also particularly vulnerable to land subsidence. India and Bangladesh are particularly at risk, in Bangladesh, a rise of $0.5 \mathrm{~m}$ would result in a loss of about $11 \%$ of the country's land, displacing approximately 15 million people.

Global warming is also accelerating desertification, with increasing number of the world's population having limited access to fresh water. African and Asian countries are the most affected by desertification, with Ethiopia, Sudan, Eritrea, Afghanistan, China, Pakistan, Iran, Somalia, Uganda and Morocco currently the top most vulnerable countries to drought, both geographically and due to lack of resilience arising from economic and socio-political factors. In addition to the increasing lack of fresh water arising from drought, pollution also threatens access to clean water. Water treatment plants are already under pressure from rising sea levels, pollution of aquifers will continue, made worse by declining streamflows; salination could also affect surfacelevel fresh water. It is estimated that more than 650 million people in 500 cities are projected to face declines in freshwater availability of at least $10 \%$ by the middle of the century.

Climate change continues to increase strain on the global food system through changes in temperature, precipitation and extreme weather events, as well as increasing $\mathrm{CO} 2$ concentrations. Climate change is affecting food security through a direct impact on agricultural output, through changing temperature and rainfall patterns, and on wider systemic disruptions such as market volatility, interruptions to transport networks, and humanitarian emergencies. Currently around $11 \%$ of the world's population suffer from undernourishment, a total of 821 million people, and the trend is currently upwards. To sustain current levels of food availability between now and 2050 will require an estimated $70 \%$ increase in food production. Climate change is also affecting food system distribution - maritime corridors, coastal infrastructure and inland transport networks. This vulnerability is compounded by the growing role of global food supply chains - between 2000 and 2015 , the volume of agricultural commodities traded internationally increased by $127 \%$.

Rapid urbanization is associated with high level of ambient air pollution, mainly caused by vehicular exhausts. A total of 3.7 million premature deaths worldwide in 2012 are attributable to outdoor air pollution. Chronic exposure to elevated levels of air pollution has been related to the incidence of chronic obstructive pulmonary disease (COPD), chronic bronchitis (CB), asthma, and emphysema. There is also growing evidence suggesting adverse effects on lung function related to long-term exposure to ambient air pollution.

Another consequence of climate change is an increase in the intensity and frequency of extreme weather events. Both the number and intensity of heat waves, floods and snow storms, gales/hurricanes/typhoons has increased. While all regions of the world are vulnerable to such events, LMICs are particularly vulnerable. Additionally these regions have a large and growing population, and increasing number of older adults.

Most research concludes that older adults face higher risks from climate change compared to the rest of the population. For example, older adults are vulnerable to being trapped in poor environments through lack of mobility, disability and frailty. 
They are at increased risk of heat-related illnesses, compounded by living alone, comorbidities, medication, and are at higher risk of dehydration than young people, due to the physiological changes that occur as part of the ageing process. Consequently any reduction in access to fresh water, during a drought for example, has severe implications for older adults. Even mild dehydration adversely affects mental performance, memory, attention, concentration and reaction time, weakness, dizziness and increased risk of falls. Kidney function, cardiac function and convulsions may follow from acute dehydration. Furthermore, drinking contaminated water leads to many diarrheal diseases including dysentery, Hepatitis E, cholera and typhoid which are frequently fatal in older people.

The low nourishment from the poor diets during a drought compounds the impact and long term implications for health. Diabetes, heart disease, depression, falls and frailty follow from even mild reduction in nutrients. In LMICs older people have a high exposure to food insecurity and a lack of access to resources for food production. They often consume low nutrient foods and contaminated foods, leading to high levels of exposure to disease and death from contamination. There is also now evidence for malnutrition among older people in many high income countries.

While as a percentage of the population, more younger than older people live in coastal cities, the numbers and percentages of older people are increasing. Older adults will be affected by an inability to make the long-term migratory moves to escape the double impact of heat and flooding in these zones. While few studies have assessed older populations specifically in terms of increased pollution, most have shown higher risks in the older adults compared to the rest of the population. This appears due to long term exposure, comorbidities and frailty increase cumulative associations between air pollution and lung function. Research has shown that older people face particular challenges during humanitarian crises bought on by climate events, including difficulty in accessing distant relief and service points. In addition most disaster response programmes lack explicit focus on older people, compounded by the view that families will provide for older members. Older populations are therefore likely to continue to be at higher risk and vulnerability with lower levels of resilience.

These environmental changes are occurring in the context of a world order which is becoming more fragile and unpredictable. The once viewed unstoppable linear progression towards greater democracy of the 1990s, with more open markets and peaceful international cooperation, appears to be weakening. Globalisation may well be driven by a new set of powers, within a new framework of understandings and values. The nature of globalisation will change, with the rise of Asia operating within different paradigms. The challenges of climate change and access to changing environmental resources will place increasing pressure on a multilateral framework developed under very different conditions. States such as Russia, Korea and some in the Middle East, will be joined by no-state players, many fuelled by religious and cultural extremism, to act as disrupters to the accepted rules of international co-operation. The potential for increased global inequality and a reduced global ability to tackle climate change will only increase the future impact on the world's older population, one of the earth's most vulnerable populations. 